\title{
B. Y. CHEN'S INEQUALITIES FOR BI-SLANT SUBMANIFOLDS IN COSYMPLECTIC SPACE FORMS
}

\author{
RAM SHANKAR GUPTA
}

\begin{abstract}
In this paper we obtain B. Y. Chen's inequalities for a bislant submanifold $\mathrm{M}$ of a cosymplectic space form $\bar{M}(\mathrm{c})$, when the structure vector field $\xi$ of the ambient space is tangent to $M$.
\end{abstract}

\section{INTRODUCTION}

In the theory of Riemannian submanifolds it is quite interesting to establish a relationship between the intrinsic and extrinsic invariants. Basically, the Riemannian invariants are intrinsic characteristics of Riemannian manifolds. In 1993, B. Y. Chen [6] has obtained an inequality between sectional curvature $\mathrm{K}$, the scalar curvature $\tau$ (intrinsic invariant) and the mean curvature function $\|H\|$ (extrinsic invariant) of a submanifold $M$ of the real space form of constant curvature $c$. Moreover, Chen [4] also introduced a new type of Riemannian invariants of a Riemannian manifold.

Let $M$ be a Riemannian manifold of dimension $m$ and let $\left\{e_{1}, e_{2}, \ldots, e_{m}\right\}$ be any orthonormal basis of the tangent space $T_{p} M$ at any point $p \in M$. then the scalar curvature $\tau$ at $p \in M$ is given by

$$
\tau=\sum_{1 \leq i<j \leq m} K\left(e_{i} \wedge e_{j}\right)
$$

for any point $p \in M$, we denote

$$
(\inf K)(p)=\inf \left\{K(\pi): \pi \subset T_{p} M, \operatorname{dim} \pi=2\right\}
$$

where $K(\pi)$ denotes the sectional curvature of $\mathrm{M}$ associated with a plane section $\pi \subset T_{p} M$ at $p \in M$.

The Chen invariant $\delta_{M}$ at any point $p \in M$ is defined as

$$
\delta_{M}(p)=\tau(p)-(\inf K)(p) .
$$

2000 Mathematics Subject Classification. 53C40, 53B25, 53D15.

Key words and phrases. Cosymplectic space form, bi-slant submanifold, slant submanifolds, mean curvature, Chen's inequality. 
For a submanifold $M$ of a real space form $\bar{M}(c)$, Chen has given a basic inequality in terms of the intrinsic invariant $\delta_{M}$ and the squared mean curvature of the immersion, as

$$
\delta_{M} \leq \frac{m^{2}(m-2)}{2(m-1)}\|H\|^{2}+\frac{1}{2}(m+1)(m-2) c .
$$

The above inequality also holds good in case $M$ is an anti-invariant submanifold of complex space form $\bar{M}(c)[7]$. In case of contact manifold, Defever, Mihai and Verstralen [11] obtained an inequality similar to that of (1.4), for C-totally real submanifold of a Sasakian space form with constant $\varphi$-sectional curvature $c$, given by

$$
\delta_{M} \leq \frac{m^{2}(m-2)}{2(m-1)}\|H\|^{2}-\frac{1}{2}(m+1)(m-2) \frac{c+3}{4} .
$$

\section{Preliminaries}

A $(2 \mathrm{~m}+1)$-dimensional Riemannian manifold $\bar{M}$ is said to be an almost contact metric manifold if there exists structure tensors $(\phi, \xi, \eta, g)$, where $\phi$ is a $(1,1)$ tensor field, $\xi$ a vector field, $\eta$ a 1 -form and $g$ the Riemannian metric on $\bar{M}$ satisfying [9]

$$
\phi^{2} X=-X+\eta(X) \xi, \quad \phi \xi=0, \quad \eta(\xi)=1, \quad \eta(\phi X)=0
$$

and

$$
g(\phi X, \phi Y)=g(X, Y)-\eta(X) \eta(Y), \quad \eta(X)=g(X, \xi)
$$

for any $X, Y \in T \bar{M}$, where $T \bar{M}$ denotes the Lie algebra of vector fields on $\bar{M}$.

An almost contact metric manifold $\bar{M}$ is called a cosymplectic manifold if [13],

$$
\left(\bar{\nabla}_{X} \phi\right) Y=0 \quad \text { and } \quad \bar{\nabla}_{X} \xi=0
$$

where $\bar{\nabla}$ denotes the Levi-Civita connection on $\bar{M}$.

The curvature tensor $\bar{R}$ of a cosymplectic space form $\bar{M}(c)$ is given by [14],

$$
\begin{aligned}
\bar{R}(X, Y) Z= & \frac{c}{4}\{g(Y, Z) X-g(X, Z) Y+\eta(X) \eta(Z) Y \\
& -\eta(Y) \eta(Z) X+\eta(Y) g(X, Z) \xi-\eta(X) g(Y, Z) \xi \\
& \quad-g(\phi X, Z) \phi Y+g(\phi Y, Z) \phi X+2 g(X, \phi Y) \phi Z\}
\end{aligned}
$$

for all $X, Y, Z \in T \bar{M}$.

Now, let $M$ be an m-dimensional isometrically immersed Riemannian submanifold of a cosymplectic manifold $\bar{M}$ with induced metric $g$. Denoting by 
$T M$ the tangent bundle of $M$ and by $T^{\perp} M$ the set of all vector fields normal to $M$, we write

$$
\phi X=P X+F X
$$

for any $X \in T M$, where $P X$ (resp. $F X$ ) denotes the tangential (resp. normal) component of $\phi X$.

From now on we assume that the structure vector field $\xi$ is tangent to $M$. We make the direct orthogonal decomposition $T M=D \oplus \xi$.

A submanifold $M$ is said to be slant if for any non zero vector $X$ tangent to $M$ at $p$ such that $X$ is not proportional to $\xi_{p}$, the angle $\theta(X)$ between $\phi X$ and $T_{p} M$ is constant i. e., is independent of the choice of $p \in M$ and $X \in T_{p} M-\left\{\xi_{p}\right\}$. Sometime the angle $\theta(X)$ is termed as the wirtinger angle of the slant immersion.

Invariant and anti-invariant immersions are slant immersions with slant angle $\theta=0$ and $\theta=\frac{\pi}{2}$, respectively. A slant immersion which is neither invariant nor anti-invariant is called a proper slant immersion.

A submanifold $M$ tangent to structure vector field $\xi$ is said to be a bi-slant submanifold of a cosymplectic manifold $\bar{M}$, if there exist two orthogonal differentiable distributions $D_{1}$ and $D_{2}$ on $M$, such that

(i) $T M$ possesses an orthogonal direct decomposition of $D_{1}$ and $D_{2}$ i. e. $T M=D_{1} \oplus D_{2} \oplus \xi$.

(ii) $D_{i}$ is slant distribution with slant angle $\theta_{i}$ for any $i=1,2$.

If we take the $\operatorname{dim} D_{1}=2 n_{1}$ and $\operatorname{dim} D_{2}=2 n_{2}$, then it is obvious that in case either $n_{1}$ vanishes or $n_{2}$, the bi-slant submanifold reduces to a slant submanifold. Hence, the bi-slant submanifolds are generalized cases of slant submanifolds. moreover, slant submanifolds, invariant submanifolds and anti-invariant submanifolds are particular cases of bi-slant submanifolds.

Let $R$ and $\bar{R}$ denote the curvature tensors of the submanifold $M$ and cosymplectic space form $\bar{M}(c)$, respectively. Then the equation of Gauss is given by

$\bar{R}(X, Y, Z, W)=R(X, Y, Z, W)-g(h(X, W), h(Y, Z))+g(h(X, Z), h(Y, W))$

for all $X, Y, Z, W \in T M$.

We denote by $h$ the second fundamental form of $M$ and by $A_{N}$ the Weingarten map associated with $N \in T^{\perp} M$. We put

$$
h_{i, j}^{r}=g\left(h\left(e_{i}, e_{j}\right), e_{r}\right) \quad \text { and } \quad\|h\|^{2}=\sum_{i, j=1}^{m} g\left(h\left(e_{i}, e_{j}\right), h\left(e_{i}, e_{j}\right)\right)
$$

for any $e_{i}, e_{j} \in T M$ and $e_{r} \in T^{\perp} M$.

The mean curvature vector $H$ is defined as $H=\frac{1}{m}$ (trace $\left.h\right)$. We say that the submanifold $M$ is minimal, if the mean curvature vector $H$ vanishes 
identically. It is well known that for a cosymplectic manifold

$$
h(X, \xi)=0 .
$$

For a given orthonormal frame $\left\{e_{1}, e_{2}, \ldots, e_{m}\right\}$ of a differentiable distribution $D$, we denote the squared norms of $P$ and $F$ respectively, by

$$
\|P\|^{2}=\sum_{i, j=1}^{m} g^{2}\left(e_{i}, P e_{j}\right) \quad \text { and } \quad\|F\|^{2}=\sum_{i=1}^{m}\left\|F e_{i}\right\|^{2} .
$$

It can be readily seen that $\|P\|^{2}$ and $\|F\|^{2}$ are independent of the choice of the above orthonormal frame.

For any $i=1,2, \ldots, m$ where $\left\{e_{1}, e_{2}, \ldots, e_{m}, \xi\right\}$ is a local orthonormal frame, we have

$$
\sum_{j=1}^{m} g^{2}\left(e_{i}, \phi e_{j}\right)=\cos ^{2} \theta .
$$

A plane section $\pi$ in a cosymplectic manifold $\bar{M}$ is said to be a $\phi$-section, if it is spanned by a unit tangent vector $X$ orthonormal to $\xi$ and $\phi X$, i. e.

$$
K(\pi)=K(X, \phi X)=g(\bar{R}(X, \phi X) \phi X, X) .
$$

The sectional curvature of a $\phi$-section is called $\phi$-sectional curvature. A cosymplectic manifold $\bar{M}$ with constant $\phi$-sectional curvature $c$ is said to be a cosymplectic space form and is usually denoted by $\bar{M}(c)$.

For an orthonormal basis $\left\{e_{1}, e_{2}, \ldots, e_{m}, e_{m+1}=\xi\right\}$ of the tangent space $T_{p} M$ at $p \in M$, from (1.1), the scalar curvature $\tau$ at $p$ of $M$ assumes the form

$$
2 \tau=\sum_{i \neq j}^{m} K\left(e_{i} \wedge e_{j}\right)+2 \sum_{i=1}^{m} K\left(e_{i} \wedge \xi\right) .
$$

Now, we mention the following results for our subsequent use.

Corollary 2.1. [12] Let $M$ be a slant submanifold of an almost contact metric manifold $\bar{M}$ with slant angle $\theta$. Then for any $X, Y \in T M$, we have

$$
\begin{aligned}
& g(P X, P Y)=\cos ^{2} \theta\{g(X, Y)-\eta(X) \eta(Y)\} \\
& g(F X, F Y)=\sin ^{2} \theta\{g(X, Y)-\eta(X) \eta(Y)\} .
\end{aligned}
$$

Lemma 2.1. [6] Let $a_{1}, a_{2}, \ldots, a_{k}, c$ be $k+1 \quad(k \geq 2)$ real numbers such that

$$
\left(\sum_{i=1}^{k} a_{i}\right)^{2}=(k-1)\left(\sum_{i=1}^{k} a_{i}^{2}+c\right) .
$$

Then $2 a_{1} a_{2} \geq c$ and the equality holds if and only if $a_{1}+a_{2}=a_{3}=\cdots=a_{k}$. 


\section{Chen's inequality for Bi-Slant submanifolds in COSympleCtiC} SPACE FORMS

Theorem 3.1. Let $\psi: M \rightarrow \bar{M}$ be an isometric immersion from a Riemannian $\left(m+1=2 n_{1}+2 n_{2}+1\right)$-dimensional bi-slant submanifold $M$ into a cosymplectic space form $\bar{M}(c)$ of dimension $2 m+1$. Then, we have

$$
\begin{aligned}
\tau-K(\pi) \leq \frac{(m+1)^{2}(m-1)}{2 m}\|H\|^{2} & +\frac{c}{8}(m+1)(m-2) \\
& +\frac{3 c}{4}\left[\left(n_{1}-1\right) \cos ^{2} \theta_{1}+n_{2} \cos ^{2} \theta_{2}\right]
\end{aligned}
$$

on $D_{1}$, and

$$
\begin{aligned}
\tau-K(\pi) \leq \frac{(m+1)^{2}(m-1)}{2 m}\|H\|^{2} & +\frac{c}{8}(m+1)(m-2) \\
& +\frac{3 c}{4}\left[n_{1} \cos ^{2} \theta_{1}+\left(n_{2}-1\right) \cos ^{2} \theta_{2}\right]
\end{aligned}
$$

on $\mathrm{D}_{2}$.

The equality cases in (3.1) and (3.2) hold at a point $p \in M$ if and only if there exist an orthonormal basis $\left\{e_{1}, e_{2}, \ldots, e_{m}, e_{m+1}=\xi\right\}$ of $T_{p} M$ and an orthonormal basis $\left\{e_{m+2}, e_{m+3}, \ldots, e_{2 m+1}\right\}$ of $T_{p}^{\perp} M$ such that the shape operators of $M$ in $\bar{M}(c)$, at a point $p$ take the following forms

$$
\begin{aligned}
& A_{m+2}=\left(\begin{array}{ccccccc}
a & 0 & 0 & \cdot & \cdot & \cdot & 0 \\
0 & b & 0 & \cdot & \cdot & \cdot & 0 \\
0 & 0 & & \lambda I_{m-1} & &
\end{array}\right), \quad a+b=\lambda \\
& A_{e_{r}}=\left(\begin{array}{ccccccc}
h_{11}^{r} & h_{12}^{r} & 0 & . & . & . & 0 \\
h_{12}^{r} & -h_{11}^{r} & 0 & . & . & . & 0 \\
0 & 0 & & & 0_{m-1} & &
\end{array}\right), \quad r \in\{m+3, \ldots, 2 m+1\}
\end{aligned}
$$

Proof. Using Gauss equation in the expression of the curvature tensor $\bar{R}$ of cosymplectic space form $\bar{M}(c)$ given by (2.3), we obtain

$$
\begin{array}{r}
R(X, Y, Z, W)=g(h(X, W), h(Y, Z))-g(h(X, Z), h(Y, W)) \\
+\frac{c}{4}\{g(Y, Z) g(X, W)-g(X, Z) g(Y, W)+\eta(X) \eta(Z) g(Y, W) \\
-\eta(Y) \eta(Z) g(X, W)+\eta(Y) \eta(W) g(X, Z)-\eta(X) \eta(W) g(Y, Z) \\
-g(\phi X, Z) g(\phi Y, W)+g(\phi Y, Z) g(\phi X, W)+2 g(X, \phi Y) g(\phi Z, W)\}
\end{array}
$$

for any $X, Y, Z, W \in T M$. 
For an orthonormal basis $\left\{e_{1}, e_{2}, \ldots, e_{m}, e_{m+1}=\xi\right\}$ of $T_{p} M$ at $p \in M$, putting $X=W=e_{i}$ and $Y=Z=e_{j}, \forall i, j \in\{1, \ldots, m+1\}$, in (3.5), we get

$$
\begin{aligned}
& \sum_{i, j=1}^{m+1} R\left(e_{i}, e_{j}, e_{j}, e_{i}\right)=g\left(h\left(e_{i}, e_{i}\right), h\left(e_{j}, e_{j}\right)\right)-g\left(h\left(e_{i}, e_{j}\right), h\left(e_{j}, e_{i}\right)\right) \\
& +\frac{c}{4}\left\{g\left(e_{j}, e_{j}\right) g\left(e_{i}, e_{i}\right)-g\left(e_{i}, e_{j}\right) g\left(e_{j}, e_{i}\right)\right\}+\frac{c}{4}\left\{\eta\left(e_{i}\right) \eta\left(e_{j}\right) g\left(e_{j}, e_{i}\right)\right. \\
& \quad-\eta\left(e_{j}\right) \eta\left(e_{j}\right) g\left(e_{i}, e_{i}\right)+\eta\left(e_{j}\right) \eta\left(e_{i}\right) g\left(e_{i}, e_{j}\right)-\eta\left(e_{i}\right) \eta\left(e_{i}\right) g\left(e_{j}, e_{j}\right) \\
& \left.-g\left(\phi e_{i}, e_{j}\right) g\left(\phi e_{j}, e_{i}\right)+g\left(\phi e_{j}, e_{j}\right) g\left(\phi e_{i}, e_{i}\right)+2 g\left(e_{i}, \phi e_{j}\right) g\left(\phi e_{j}, e_{i}\right)\right\}
\end{aligned}
$$

or,

$$
\begin{gathered}
\sum_{i, j=1}^{m+1} R\left(e_{i}, e_{j}, e_{j}, e_{i}\right)=(m+1)^{2}\|H\|^{2}-\|h\|^{2}+\frac{c}{4}\left\{(m+1)^{2}-(m+1)\right\} \\
+\frac{c}{4}\left\{1-(m+1)+1-(m+1)+3 \sum_{i, j=1}^{m+1} g^{2}\left(e_{i}, \phi e_{j}\right)\right\}
\end{gathered}
$$

or,

$$
\begin{gathered}
\sum_{i \neq j}^{m} R\left(e_{i}, e_{j}, e_{j}, e_{i}\right)+2 \sum_{i=1}^{m} R\left(e_{i}, \xi, \xi, e_{i}\right)=(m+1)^{2}\|H\|^{2}-\|h\|^{2} \\
+\frac{c}{4}\left\{(m+1)^{2}-(m+1)\right\}+\frac{c}{4}\left\{-2 m+3 \sum_{i, j=1}^{m+1} g^{2}\left(e_{i}, \phi e_{j}\right)\right\} .
\end{gathered}
$$

Now using (2.11) in the above equation, we get

$$
2 \tau=(m+1)^{2}\|H\|^{2}-\|h\|^{2}+\frac{c}{4} m(m+1)+\frac{c}{4}\left\{-2 m+3 \sum_{i, j=1}^{m+1} g^{2}\left(e_{i}, \phi e_{j}\right)\right\}
$$

or,

$$
2 \tau=(m+1)^{2}\|H\|^{2}-\|h\|^{2}+\frac{c}{4} m(m-1)+3 \frac{c}{4} \sum_{i, j=1}^{m+1} g^{2}\left(e_{i}, \phi e_{j}\right) .
$$

Since $M^{m+1}$ is bi-slant submanifold of a cosymplectic space form $\bar{M}^{2 m+1}(c)$, where $(m+1)=2 n_{1}+2 n_{2}+1$, we may consider an adapted bi-slant orthonormal frame as follows:

$$
\begin{gathered}
e_{1}, e_{2}=\sec \theta_{1} P e_{1}, \ldots, e_{2 n_{1}-1}, e_{2 n_{1}}=\sec \theta_{1} P e_{2 n_{1}-1} \\
e_{2 n_{1}+1}, e_{2 n_{1}+2}=\sec \theta_{2} P e_{2 n_{1}+1}, \ldots, e_{2 n_{1}+2 n_{2}-1}, e_{2 n_{1}+2 n_{2}} \\
=\sec \theta_{2} P e_{2 n_{1}+2 n_{2}-1} \text { and } e_{2 n_{1}+2 n_{2}+1}=\xi .
\end{gathered}
$$


Then, we have

$$
g\left(e_{1}, \phi e_{2}\right)=-g\left(\phi e_{1}, e_{2}\right)=-g\left(\phi_{1}, \sec \theta_{1} P e_{1}\right)
$$

or,

$$
g\left(e_{1}, \phi e_{2}\right)=-\sec \theta_{1} g\left(P e_{1}, P e_{1}\right) .
$$

Now, using (2.12), we get

$$
g\left(e_{1}, \phi e_{2}\right)=-\cos \theta_{1}
$$

or,

$$
g^{2}\left(e_{1}, \phi e_{2}\right)=\cos ^{2} \theta_{1}
$$

Similarly,

$$
g^{2}\left(e_{i}, \phi e_{i+1}\right)= \begin{cases}\cos ^{2} \theta_{1}, & \text { for } i=1, \ldots, 2 n_{1}-1 \\ \cos ^{2} \theta_{2}, & \text { for } i=2 n_{1}+1, \ldots, 2 n_{1}+2 n_{2}-1 .\end{cases}
$$

Hence, we have

$$
\sum_{i, j=1}^{m+1} g^{2}\left(e_{i}, \phi e_{j}\right)=2\left\{n_{1} \cos ^{2} \theta_{1}+n_{2} \cos ^{2} \theta_{2}\right\} .
$$

Using this relation in (3.6), we obtain

$$
2 \tau=(m+1)^{2}\|H\|^{2}-\|h\|^{2}+\frac{c}{4} m(m-1)+\frac{3 c}{4}\left[2\left(n_{1} \cos ^{2} \theta_{1}+n_{2} \cos ^{2} \theta_{2}\right)\right] .
$$

Putting

$\epsilon=2 \tau-\frac{(m+1)^{2}(m-1)}{m}\|H\|^{2}-\frac{c}{4}(m+1)(m-2)-\frac{3 c}{2}\left[n_{1} \cos ^{2} \theta_{1}+n_{2} \cos ^{2} \theta_{2}\right]$

in (3.7), we get

or,

$$
\epsilon=\frac{(m+1)^{2}}{m}\|H\|^{2}-\|h\|^{2}+\frac{c}{2}
$$

$$
(m+1)^{2}\|H\|^{2}=m\|h\|^{2}+m\left\{\epsilon-\frac{c}{2}\right\} .
$$

Let $p \in M, \pi \subset T_{p} M, \operatorname{dim} \pi=2$ and $\pi$ is orthogonal to $\xi$.

Now, we consider the following two cases:

Case (i). Let $\pi$ be tangent to the differentiable distribution $D_{1}$ and let it be spanned by orthonormal basis vectors $e_{1}$ and $e_{2}$. If we take $e_{m+2}$ in the direction of mean curvature vector $H$ i. e. $e_{m+2}=\frac{H}{\|H\|}$, then from (3.9), we get

$$
\left(\sum_{i=1}^{m+1} h_{i i}^{m+2}\right)^{2}=m\left\{\sum_{i=1}^{m+1}\left(h_{i i}^{m+2}\right)^{2}+\sum_{i \neq j}\left(h_{i j}^{m+2}\right)^{2}+\sum_{r=m+3}^{2 m+1} \sum_{i, j}\left(h_{i j}^{r}\right)^{2}+\epsilon-\frac{c}{2}\right\} .
$$


Now using lemma (2.1) in (3.10), we get

$$
2 h_{11}^{m+2} h_{22}^{m+2} \geq \sum_{i \neq j}\left(h_{i j}^{m+2}\right)^{2}+\sum_{r=m+3}^{2 m+1} \sum_{i, j}\left(h_{i j}^{r}\right)^{2}+\epsilon-\frac{c}{2} .
$$

On the other hand, we have

$$
\begin{aligned}
K(\pi)=R\left(e_{1}, e_{2}, e_{2}, e_{1}\right)=g\left(h\left(e_{1}, e_{1}\right), h\left(e_{2}, e_{2}\right)\right) & \\
& -g\left(h\left(e_{1}, e_{2}\right), h\left(e_{1}, e_{2}\right)\right)+\frac{c}{4}+\frac{3 c}{4} \cos ^{2} \theta_{1}
\end{aligned}
$$

or,

$$
\begin{aligned}
& K(\pi)=\sum_{r=m+2}^{2 m+1}\left\{g\left(h\left(e_{1}, e_{1}\right), e_{r}\right) g\left(h\left(e_{2}, e_{2}\right), e_{r}\right)\right. \\
&\left.\quad-g\left(h\left(e_{1}, e_{2}\right), e_{r}\right) g\left(h\left(e_{1}, e_{2}\right), e_{r}\right)+\frac{c}{4}+\frac{3 c}{4} \cos ^{2} \theta_{1}\right\}
\end{aligned}
$$

or,

$$
K(\pi)=\sum_{r=m+2}^{2 m+1}\left\{h_{11}^{r} h_{22}^{r}-\left(h_{12}^{r}\right)^{2}\right\}+\frac{c}{4}+3 \frac{c}{4} \cos ^{2} \theta_{1}
$$

or,

$$
K(\pi)=h_{11}^{m+2} h_{22}^{m+2}+\sum_{r=m+3}^{2 m+1} h_{11}^{r} h_{22}^{r}-\sum_{r=m+2}^{2 m+1}\left(h_{12}^{r}\right)^{2}+\frac{c}{4}+\frac{3 c}{4} \cos ^{2} \theta_{1} .
$$

Using (3.11) in the above equation, we obtain

$$
\begin{aligned}
k(\pi) \geq \frac{1}{2} \sum_{i \neq j} & \left(h_{i j}^{m+2}\right)^{2}+\frac{1}{2} \sum_{r=m+3}^{2 m+1} \sum_{i j=1}^{2 m+1}\left(h_{i j}^{r}\right)^{2} \\
& +\frac{\epsilon}{2}-\frac{c}{4}+\sum_{r=m+3}^{2 m+1} h_{11}^{r} h_{22}^{r}-\sum_{r=m+2}^{2 m+1}\left(h_{12}^{r}\right)^{2}+\frac{c}{4}+\frac{3 c}{4} \cos ^{2} \theta_{1}
\end{aligned}
$$

or,

$$
K(\pi) \geq \frac{\epsilon}{2}+3 \frac{c}{4} \cos ^{2} \theta_{1} .
$$

Now using (3.8) in (3.13), we obtain

$$
\begin{array}{r}
\tau-K(\pi) \leq \frac{(m+1)^{2}(m-1)}{2 m}\|H\|^{2}+\frac{c}{8}(m+1)(m-2) \\
+\frac{3 c}{4}\left[\left(n_{1}-1\right) \cos ^{2} \theta_{1}+n_{2} \cos ^{2} \theta_{2}\right] .
\end{array}
$$


Case (ii). If $\pi$ is tangent to $D_{2}$, we obtain, as in Case (i)

$$
\begin{aligned}
\tau-K(\pi) \leq \frac{(m+1)^{2}(m-1)}{2 m} & \|H\|^{2}+\frac{c}{8}(m+1)(m-2) \\
+ & \frac{3 c}{4}\left[n_{1} \cos ^{2} \theta_{1}+\left(n_{2}-1\right) \cos ^{2} \theta_{2}\right] .
\end{aligned}
$$

These are the desired inequalities.

If at any point $p \in M$, equality in (3.1) and (3.2) hold, then the inequalities in (3.11) and (3.13) become equalities. Hence, we have

$$
\begin{gathered}
h_{1 j}^{m+2}=h_{2 j}^{m+2}=h_{i j}^{m+2}=0, \quad i \neq j>2 \\
h_{i j}^{r}=0, \quad \forall i \neq j, \quad i, j=3, \ldots, 2 m+1, r=m+3, \ldots, 2 m+1 \\
h_{11}^{r}+h_{22}^{r}=0, \quad \forall r=m+3, \ldots, 2 m+1 \\
h_{11}^{m+2}+h_{22}^{m+2}=h_{33}^{m+2}=\cdots=h_{m+1 m+1}^{m+2} .
\end{gathered}
$$

Now, if we take $e_{1}, e_{2}$ such that $h_{12}^{m+2}=0$ and letting $a=h_{11}^{r}, b=$ $h_{22}^{r}, \lambda=h_{33}^{m+2}=\cdots=h_{m+1 m+1}^{m+2}$, it follows that the shape operators assume the desired form.

Corollary 3.1. Let $M$ be an $m+1$-dimensional contact $C R$-submanifold with in a $2 m+1$-dimensional cosymplectic space form $\bar{M}(c)$. Then, we have

$$
\tau-K(\pi) \leq \frac{(m+1)^{2}(m-1)}{2 m}\|H\|^{2}+\frac{c}{8}(m+1)(m-2)+\frac{3 c}{4}\left(n_{1}-1\right)
$$

on $D_{1}$, and

$$
\tau-K(\pi) \leq \frac{(m+1)^{2}(m-1)}{2 m}\|H\|^{2}+\frac{c}{8}(m+1)(m-2)+\frac{3 c}{4} n_{1}
$$

on $\mathrm{D}_{2}$.

Now, we have the following result.

Theorem 3.2. Let $M$ be an $(m+1)$-dimensional $\theta$-slant submanifold with $\theta_{1}=\theta_{2}=\theta$ in $a(2 m+1)$-dimensional cosymplectic space form $\bar{M}(c)$. Then, we have

$$
\delta_{M} \leq \frac{(m+1)^{2}(m-1)}{2 m}\|H\|^{2}+\frac{c}{8}(m+1)(m-2)+\frac{3 c}{8}(m-2) \cos ^{2} \theta .
$$

The equality holds at a point $p \in M$ if and only if there exists an orthonormal basis $\left\{e_{1}, e_{2}, \ldots, e_{m}, e_{m+1}=\xi\right\}$ of $T_{p} M$ and an orthonormal basis $\left\{e_{m+2}, e_{m+3}, \ldots, e_{2 m+1}\right\}$ of $T_{p}^{\perp} M$ such that the shape operators of $M$ 
in cosymplectic space form $\bar{M}(c)$ take the following forms

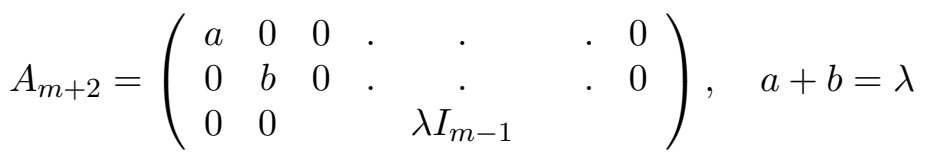

$$
\begin{aligned}
& A_{e_{r}}=\left(\begin{array}{ccccccc}
h_{11}^{r} & h_{12}^{r} & 0 & . & . & . & 0 \\
h_{12}^{r} & -h_{11}^{r} & 0 & . & . & . & 0 \\
0 & 0 & & & 0
\end{array}\right), \quad r=m+3, \ldots, 2 m+1 .
\end{aligned}
$$

Corollary 3.2. Let $M$ be an $(m+1)$-dimensional invariant submanifold of $a(2 m+1)$-dimensional cosymplectic space form $\bar{M}(c)$. Then, we have

$$
\delta_{M} \leq \frac{c\left(m^{2}+2 m-8\right)}{8} .
$$

Corollary 3.3. Let $M$ be an $(m+1)$-dimensional anti-invariant submanifold of a $(2 m+1)$-dimensional cosymplectic space form $\bar{M}(c)$. Then, we have

$$
\delta_{M} \leq \frac{(m+1)^{2}(m-1)}{2 m}\|H\|^{2}+\frac{c}{8}(m+1)(m-2) .
$$

4. EXAMPLES OF BI-SLANT SUBMANIFOLDS OF COSYMPLECTIC

$$
\text { MANIFOLDS }
$$

Example 4.1. For any $\theta_{1}, \theta_{2} \in[0, \pi / 2]$

$$
x(u, v, w, s, z)=\left(u, 0, w, 0, v \cos \theta_{1}, v \sin \theta_{1}, s \cos \theta_{2}, s \sin \theta_{2}, z\right)
$$

defines a 5 -dimensional bi-slant submanifold $M$, with slant angles $\theta_{1}$ and $\theta_{2}$ in $R^{9}$ with its usual cosymplectic structure $\left(\phi_{0}, \xi, \eta, g\right)$, given by:

$$
\begin{gathered}
\eta=d z, \quad \xi=\frac{\partial}{\partial z} \\
g=\eta \otimes \eta+\left\{\sum_{i=1}^{4}\left(d x^{i} \otimes d x^{i}+d y^{i} \otimes d y^{i}\right)\right\}
\end{gathered}
$$

and

$$
\phi_{0}\left\{\sum_{i=1}^{4}\left(X_{i} \frac{\partial}{\partial x^{i}}+Y_{i} \frac{\partial}{\partial y^{i}}\right)+Z \frac{\partial}{\partial z}\right\}=\sum_{i=1}^{4}\left(-Y_{i} \frac{\partial}{\partial x^{i}}+X_{i} \frac{\partial}{\partial y^{i}}\right) .
$$

Furthermore it is easy to see that:

$$
\begin{gathered}
e_{1}=\frac{\partial}{\partial x^{1}}, \quad e_{2}=\cos \theta_{1} \frac{\partial}{\partial y^{1}}+\sin \theta_{1} \frac{\partial}{\partial y^{2}}, \quad e_{3}=\frac{\partial}{\partial x^{3}} \\
e_{4}=\cos \theta_{2} \frac{\partial}{\partial y^{3}}+\sin \theta_{1} \frac{\partial}{\partial y^{4}} \quad \text { and } \quad e_{5}=\frac{\partial}{\partial z}=\xi
\end{gathered}
$$


form a local orthonormal frame of $T M$. If, we define $D_{1}=\left\{e_{1}, e_{2}\right\}$ and $D_{2}=\left\{e_{3}, e_{4}\right\}$, then a simple computation yields, $g\left(\phi_{0} e_{1}, e_{2}\right)=\cos \theta_{1}$ and $g\left(\phi_{0} e_{3}, e_{4}\right)=\cos \theta_{2}$ proving that the distribution $D_{1}$ is $\theta_{1}$-slant and the distribution $D_{2}$ is $\theta_{2}$-slant.

Example 4.2. For any $\theta_{1}, \theta_{2} \in[0, \pi / 2]$

$$
\begin{aligned}
& x(u, v, w, s, z)=\left(\cos \alpha_{1} \cos \alpha_{2} u-\sin \alpha_{1} s, \sin \alpha_{1} \cos \alpha_{2} u\right. \\
& \left.\quad+\cos \alpha_{1} s, \cos \alpha_{1} \sin \alpha_{2} u, \sin \alpha_{1} \sin \alpha_{2} u, w,-\sin \alpha_{2} v, 0, \cos \alpha_{2} v, z\right)
\end{aligned}
$$

defines a 5-dimensional bi-slant submanifold $M$, with slant angles $\theta_{1}=\pi / 2$ and $\cos ^{2} \theta_{2}=\sin ^{2} \alpha_{1}$ in $R^{9}$ with its usual cosymplectic structure.

We can choose orthonormal frame on $T M$, given by

$e_{1}=\left(\cos \alpha_{1} \cos \alpha_{2}, \sin \alpha_{1} \cos \alpha_{2}, \cos \alpha_{1} \sin \alpha_{2}, \sin \alpha_{1} \sin \alpha_{2}, 0,0,0,0,0\right)$

$$
\begin{gathered}
e_{2}=-\sin \alpha_{2} \frac{\partial}{\partial y^{2}}+\cos \alpha_{2} \frac{\partial}{\partial y^{4}}, \quad e_{3}=\frac{\partial}{\partial y^{1}} \\
e_{4}=-\sin \alpha_{1} \frac{\partial}{\partial x^{1}}+\cos \alpha_{1} \frac{\partial}{\partial x^{2}} \quad \text { and } \quad e_{5}=\frac{\partial}{\partial z}=\xi
\end{gathered}
$$

where, distributions are defined by $D_{1}=\left\{e_{1}, e_{2}\right\}$ and $D_{2}=\left\{e_{3}, e_{4}\right\}$. Then it can be easily seen that $g\left(e_{1}, \phi_{0} e_{2}\right)=0$ and $g\left(e_{3}, \phi_{0} e_{4}\right)=\sin \alpha_{1}$, that is, distribution $D_{1}$ is $\theta_{1}$-slant with $\theta_{1}=\pi / 2$ and the distribution $D_{2}$ is $\theta_{2}$-slant with $\cos ^{2} \theta_{2}=\sin ^{2} \alpha_{1}$.

Acknowledgement. I would like to thank referee for careful reading of the manuscript and pointing out the mistakes.

\section{REFERENCES}

[1] A. Carriazo: A contact version of B. Y. Chen's inequality and its applications to slant immersions, Kyungpook Math., J. 39 (1999), 465-476.

[2] A. Lotta, Slant submanifolds in contact geometry, Bull. Math. Soc. Roumanie, 39 (1996), 183-198.

[3] B. Y. Chen, A general inequality for submanifolds in complex space forms and its applications, Arch. Math., 67 (1996), 519-528.

[4] B. Y. Chen, A Riemannian invariant and its applications to submanifold theory, Result. Math., 27 (1995), 17-26.

[5] B. Y. Chen, Geometry of Slant Submanifolds, Katholieke universiteit Leuven, 1990.

[6] B. Y. Chen, Some pinching and classification theorems for minimal submanifolds, Arch. Math., 60 (1993), 568-578.

[7] B. Y. Chen, F. Dillen, L. Verstraelen and L. Vrancken, An exotic totally real minimal immersion of $S^{3}$ in $P^{3}$ and its characterization, Proc. Roy. Soc. Edinburgh Sect. A, 126 (1996), 153-165.

[8] D. Cioroboiu, B. Y. Chen inequalities for bi-slant submanifolds in sasakian space forms, Demonstratio Math., 38 (1) (2003), 179-187. 
[9] D. E. Blair, Contact Manifolds in Riemannian Geometry, Lect. Notes in Math. Springer Verlag, Berlin- New York, 509, 1976.

[10] D. Cioroboiu abd A. Oiaga, B.Y. Chen inequalities for slant submanifolds in Sasakian space forms, Rend. Circ. Sem. Mat. Palermo, 52 (2) (2003), 367-381.

[11] F. Defever, I. Mihai, and L. Verstraelen, B. Y. Chens inequality for C-totally real submanifolds of Sasakian space forms, Bollettino U. M. I., 11 (7) (1997), 365-374.

[12] J. L. Cabrerizo, A. Carriazo, L. M. Fernandez and M. Fernandez, Slant submanifolds in Sasakian manifolds, Glasgow Math. J., 42 (2000), 125-138.

[13] G. D. Ludden, Submanifolds of cosymplectic manifolds, J. Differ. Geom., 4 (1970), $237-244$

[14] M. Kobayashi, Semi-invariant submanifolds of a certain class of almost contact manifolds, Tensor (N. S.), 43 (1986), 28-36.

[15] Ram Shankar Gupta, S. M. Khursheed Haider and M. H. Sahid, Slant submanifolds of cosymplectic manifolds, An. St. Univ. AI. I. Cuza, 50 (1) (2004), 33-49.

(Received: January 5, 2012)

(Revised: May 9, 2012)
University School of Basic and Applied Sciences Guru Gobind Singh Indraprastha University Sector-16C, Dwarka, New Delhi-110075 India

E-mail: ramshankar.gupta@gmail.com 